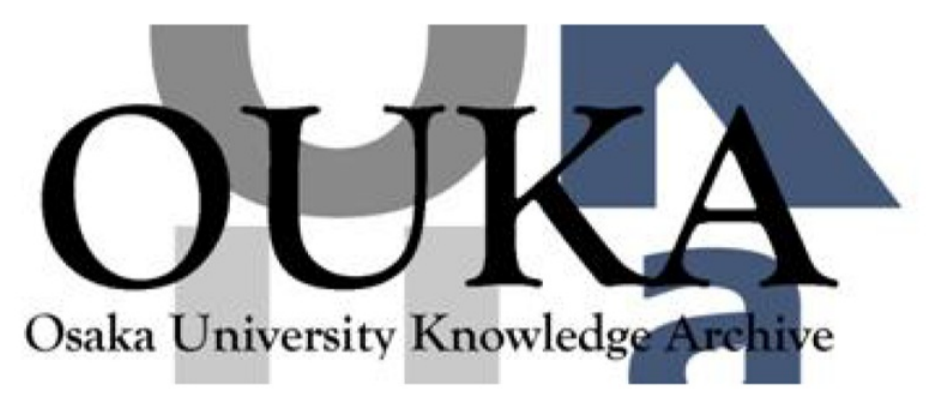

\begin{tabular}{|c|l|}
\hline Title & $\begin{array}{l}\text { Development of multi-channel electron } \\
\text { spectrometer }\end{array}$ \\
\hline Author(s) & Iwawaki, T.; Habara, H. ; Tanimoto, T. et al. \\
\hline Citation & $\begin{array}{l}\text { Review of Scient ific Instruments. 81(10) } \\
\text { p.10E535-1-p. 10E535-3 }\end{array}$ \\
\hline Issue Date & $2010-01$ \\
\hline oaire:version & VoR \\
\hline URL & https://hdl. handle. net/11094/3329 \\
\hline rights & \\
\hline Note & \\
\hline
\end{tabular}

Osaka University Knowledge Archive : OUKA

https://ir. Library. osaka-u. ac. jp/

Osaka University 


\title{
Development of multi-channel electron spectrometer ${ }^{a)}$
}

\author{
T. Iwawaki, ${ }^{1,2}$ H. Habara, ${ }^{1,2, b)}$ T. Tanimoto, ${ }^{3}$ N. Nakanii, ${ }^{1,2}$ K. Shimada, ${ }^{1,2}$ T. Yabuuchi, ${ }^{4}$ \\ K. Kondo, ${ }^{3}$ and K. A. Tanaka ${ }^{1,2}$ \\ ${ }^{1}$ Graduate School of Engineering, Osaka University, Yamada-oka, 2-1, Suita, Osaka 565-0871, Japan \\ ${ }^{2}$ Institute of Laser Engineering, Osaka University, Yamada-oka, 2-6, Suita, Osaka 565-0871, Japan \\ ${ }^{3}$ Kansai Photon Science Institute, Japan Atomic Energy Agency, Umemidai, 8-1-7, Kizugawa, Kyoto, Japan \\ ${ }^{4}$ Center for Energy Research, University of California-San Diego, 9500 Gillman Drive 0417, La Jolla, \\ California 92093-0417, USA
}

(Presented 20 May 2010; received 17 May 2010; accepted 26 July 2010;

published online 27 October 2010)

\begin{abstract}
In order to obtain the angular dependent electron energy distributions, we developed a multichannel electron spectrometer (MCESM) with high energy and angular resolutions. The MCESM consists of seven small electron spectrometers set in every $5^{\circ}$ on the basement, each of which detection range is up to $25 \mathrm{MeV}$. In the experiment, we successfully obtained electron spectra from imploded cone-shell target as well as gold plane target irradiated by ultraintense (300 J/5 ps) laser beam.

(C) 2010 American Institute of Physics. [doi:10.1063/1.3485102]
\end{abstract}

\section{INTRODUCTION}

In the fast ignition (FI) of inertial fusion research, an ultraintense laser (UIL) pulse is irradiated on the imploded plasma in order to generate hot electrons to heat the highdensity core plasma. At the time, the measurement of fast electrons (number, slope temperature, emission angle, and so on) is crucially important for estimation of heating efficiency to the core. In the previous integrated experiment at Osaka University, we observed that fast electron spectra show significant absorption at the high-density core plasma only taken at optimum injection timing of UIL. ${ }^{1}$ However the spectrum for the laser off-axis did not exhibit such modification in any timing. This result strongly indicates that the spatial resolved electron spectra can infer the spatial information of the core such as heated size or temperature. From this viewpoint, we developed a multi-channel electron spectrometer (MCESM) with a high angular resolution.

\section{MAGNET ELECTRON SPECTROMETER (ESM)}

The magnet electron spectrometer is one of the simple diagnostics to measure the energy of electrons. If the magnetic flux density can be considered as a constant, the electrons move according to a simple Larmor motion only determined by the electron kinetic energy. Because different energy electrons reach on the different positions in the detector, the electron energy distribution can be easily obtained. The energy on the position $l$ can be given by

$$
\varepsilon=m_{0} c\left[\sqrt{\left(\frac{l^{2}+h^{2}}{2 h}\right)^{2}\left(\frac{e B}{m_{0} c}\right)^{2}+1}-1\right] .
$$

Here, $h$ is the height from the electron incidence to detector, $\varepsilon$ is the electron energy, $e$ is the electric charge, $B$ is the

\footnotetext{
${ }^{a}$ Contributed paper, published as part of the Proceedings of the 18th Topical Conference on High-Temperature Plasma Diagnostics, Wildwood, New Jersey, May 2010.

b)Electonic mail: habara@eei.eng.osaka-u.ac.jp.
}

magnetic flux density, $m_{0}$ is the mass of electron, and $c$ is the speed of light, respectively.

Because fast electrons created by UIL have considerable large emission angles, a collimator is used to guarantee a parallel electron beam into the magnetic field. Consequently, electron spectrum per solid angles and per energy can be given as

$\frac{d^{2} N}{d \Omega d \varepsilon}=\frac{1}{\Delta l \Delta \Omega}\left(\frac{I}{\zeta(\varepsilon) \varphi}\right) \frac{h}{l} \frac{1}{c e B} \sqrt{1+\left(\frac{2 h}{l^{2}+h^{2}}\right)^{2}\left(\frac{m_{0} c}{e B}\right)^{2}}$,

where $N$ is the number of electrons, $I$ is the signal intensity, $\Delta l$ is the spatial resolution of the imaging plate (IP), $\Delta \Omega$ are the solid angles, $\zeta(\varepsilon)$ is the detector sensitivity, and $\varphi$ is the fading rate. ${ }^{2}$

As an electron detector, we use an IP that has a large dynamic range $\left(\sim 10^{5}\right)$ and a high sensitivity $(\sim 100$ times higher than normal $\mathrm{x}$-ray film). In addition IP is free from strong electromagnetic pulse noises created by UIL interaction with target. ${ }^{2}$

\section{DESIGN OF MCESM}

\section{A. Required specifications}

In this section, we consider a required range and resolution on angle and energy for MCESM. In the previous FI experiment, ${ }^{1}$ the core diameter is about $50 \mu \mathrm{m}$ and the distance from the center of core plasma to the gold cone tip is also $50 \mu \mathrm{m}$. From this geometry, it is expected that the electrons within $30^{\circ}$ divergence angles (half angle) are possible to interact with the core. We therefore decide that the angular range must be about $30^{\circ}$ at least with $5^{\circ}$ angular resolutions. On the other hand, the modification on electron spectrum is mostly appeared within $10 \mathrm{MeV}$ at the previous experiment. So the energy range is set from 0.2 to $25 \mathrm{MeV}$ with high energy resolution (it is about $50 \mathrm{keV}$ at $10 \mathrm{MeV}$ ).

\section{B. Mechanical design}

We designed the MCESM for the Gekko FIREX experiment. ${ }^{3}$ The diameter of the Gekko chamber is $2 \mathrm{~m}$ and 


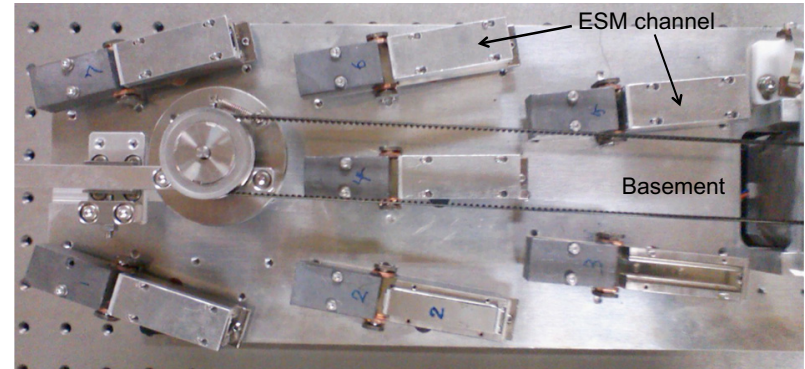

FIG. 1. (Color online) MCESM overview.

the nearest port angle is $20^{\circ}$. It is therefore necessary to insert the spectrometer to the chamber and bring it close to target chamber center (TCC) in order to obtain the required angular resolution.

Due to the restriction of the solid angle of one chamber port, we decided to cover $35^{\circ}$ angular ranges with about $20 \mathrm{~cm}$ distance from TCC. MCESM is translated close to TCC via motorized rails. The head of MCESM has a mock-up shell target that can move in and out inside the vacuum chamber. By adjusting the target in the optimum position using the target monitor system, MCESM can be assured to set the exact position with the error less than $100 \mu \mathrm{m}$ in three dimensions.

\section{Collimator design}

In order to obtain the well-collimated electron beam to the ESM, we use a thick lead block with a small diameter collimator. The thickness of the block is determined by using the continuous-slowing-down approximation (CSDA) range to stop enough high-energy electrons. Because the electrons generated at the fast ignition experiment have the kinetic energy up to several hundred $\mathrm{MeV}$, we chose a $30 \mathrm{~mm}$ length lead that can stop the electrons up to $1 \mathrm{GeV}$ as well as $\mathrm{x}$ rays from the target itself.

On the other hand, the collimator size conflictingly affects the errors on energy and number. If the collimator size is large, electrons cannot be considered as a parallel beam, resulting in a significant error on the determination of electron energy from the signal position on IP. On the other hand, the smaller diameter loses tolerance on the alignment error, which significantly reduces the detected number of electrons as described in Sec. IV C 2. Here, we referred to our single channel ESM, ${ }^{2}$ which has $1 \times 10^{-5}$ sr. Taking account of the distance from TCC, the collimator diameter is set about $700 \mu \mathrm{m}$.

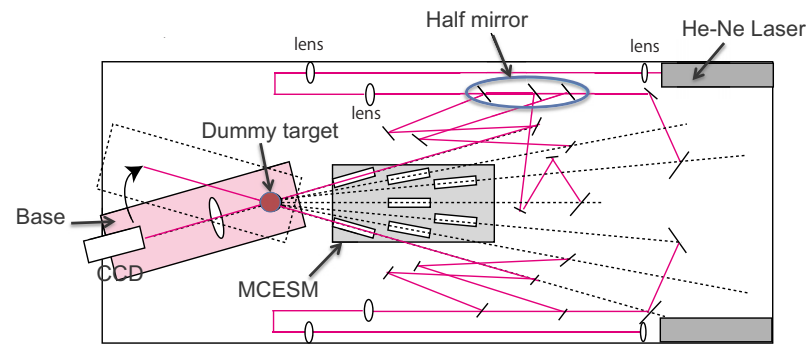

FIG. 2. (Color online) Detector alignment method with $\mathrm{He}-\mathrm{Ne}$ laser. Each light path passing through each of the ESM channel irradiates the mock-up target at virtual TCC position.

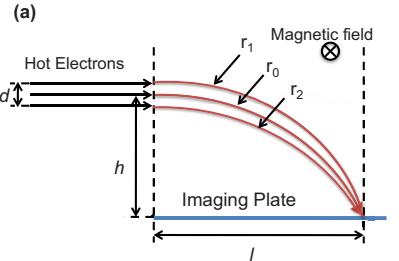

(b)

FIG. 3. (Color online) (a) Different orbits on the same IP position due to finite collimator size. (b) Electron number reduction due to tilting misalignment.

\section{DEVELOPMENT OF MULTICHANNEL ESM}

\section{A. Arrangement of ESM channels}

Figure 1 shows seven ESM channels arranged on the basement with $5^{\circ}$ angular intervals with different distance from 15.5 to $33.0 \mathrm{~cm}$. The magnetic flux density is $0.45 \mathrm{~T}$ and the magnet length is $40 \mathrm{~mm}$. The collimator is set in the front side of the magnet pair. The total length is $75 \mathrm{~mm}$ including a gap between magnets and collimator. IP is set on the top of the magnets with cover holder parallel to the electron beam.

\section{B. Method of alignment}

It is important to align each ESM channel exactly toward TCC. Because the collimator diameter is very small, even a little misalignment causes a large error on the electron number. As shown in Fig. 2, the head of MCESM needs to prealign at outside of the chamber. Two He-Ne laser lights are divided into seven paths for each channel. All laser lights simultaneously irradiate on the mock-up target equipped with the basement. The laser light is detected with charge coupled device (CCD) at the back of the target. Then each channel is put in each laser path and aligned to obtain an image that the diameter of laser becomes largest (about $640 \mu \mathrm{m})$ and a shadow of target comes in the center of laser light.

\section{Error estimation}

\section{Electron energy}

Even electron beam is injected parallel to the collimator, the error on electron energy depends on the collimator size and height to IP, $h$, in Eq. (1). As shown in Fig. 3(a), different energy electrons coming from the top (bottom) of collimator reach as same position on IP through the orbit $\mathrm{r} 1$ ( $\mathrm{r} 2$ ) as the center part of electrons. From this consideration, we choose the collimator size and $h$ in order that maximum error on energy of electrons becomes less than 5\%.

\section{Electron number}

The electron number is proportional to the cross section when electrons pass the collimator. If ESM is aligned correctly, the cross section is same as the collimator size. However, if it is slightly tilted or rotated, the collimator clips electron beam, resulting in reduction of number of detected electrons as shown in Fig. 3(b). Especially for MCESM, because each ESM channel has a freedom in three dimensions individually, the relative error loses reliability for angular distribution of fast electrons. Taking account of the off-line 

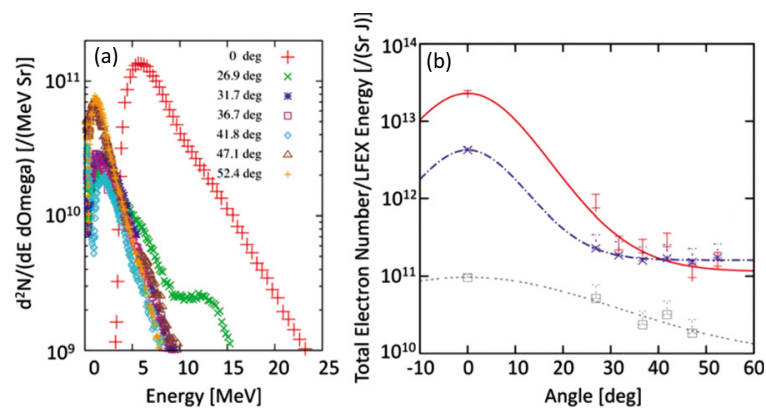

FIG. 4. (Color online) (a) Full set of angular resolved spectra taken at FI experiment. (b) Integrated total electron number normalized by heating laser energy. Open squares represent the results from gold foil target, and other symbols are taken at fast ignition shots.

alignment method above, the maximum reduction on number is estimated at about $15 \%$. The error due to setting accuracy of the mock-up target in TCC can be negligible because the collimator size is much larger than the core size $(\sim 50 \mu \mathrm{m})$.

\section{Viewing angle}

The angular error is caused by errors from the mechanical channel position and the setting accuracy of the mock-up target in TCC. The mechanical setting accuracy is $100 \mu \mathrm{m}$ in three dimensions and the setting accuracy of the target in TCC is $100 \mu \mathrm{m}$ as mentioned above. Consequently, we can estimate the error concerning the viewing angle that is about $0.07^{\circ}$ as a maximum.

\section{Noise reduction}

We performed a preliminary experiment using a plane target. In the result, the signals on IP were not so clear due to the strong noise spreading in whole area, resulting in the $\mathrm{S} / \mathrm{N}$ (signal divided by noise) ratio of $\sim 1$. Because the $\mathrm{x}$ ray or electrons from the target must be almost stopped in the lead blocks and IP is set to parallel to the electron beam axis to minimize such X-ray exposure from the target, we suspect that the source of this noise is $\mathrm{x}$ - or $\gamma$-rays created via interaction of electrons with the neighbor ESM channel or basement. In order to reduce the noise to improve $\mathrm{S} / \mathrm{N}$ ratio, we covered each ESM with $2 \mathrm{~mm}$ thickness lead sheet. Using the shield, the $\mathrm{S} / \mathrm{N}$ ratio was improved to be 8 .

\section{FAST IGNITION EXPERIMENT}

We measured the angular dependent electron spectra with the MCESM and single channel ESM at fast ignition integrated experiment conducted in Osaka University. The detector angles are $0^{\circ}, 26.9^{\circ}, 31.7^{\circ}, 36.7^{\circ}, 41.8^{\circ}, 47.1^{\circ}$, $52.4^{\circ}$, and $57.9^{\circ}$ from the laser axis, respectively. Typical result is shown in Fig. 4(a). The spectra are taken at the shot when heating laser energy is $310 \mathrm{~J}$ with 5 ps pulse duration. The target is gold cone shell. The shell diameter is $500 \mu \mathrm{m}$ and the cone tip diameter is $10 \mu \mathrm{m}$ with $10 \mu \mathrm{m}$ thickness. Implosion energy is $2.9 \mathrm{~kJ}$. The intense laser beam is injected at the timing of maximum implosion. At this shot the neutron yield is $1 \times 10^{4}$. Unfortunately in most of experiments, electron spectrum was not obtained at $57.9^{\circ}$ due to poor $\mathrm{S} / \mathrm{N}$ ratio.

Figure 4(b) shows a summary of the beam divergence for different target conditions. The vertical axis represents the number of electrons normalized by heating laser energy. Moreover, the horizontal one is the observed angle. We calculate the total electron number by fitting the observed spectrum with Eq. (3),

$$
N(\varepsilon)=\frac{N_{0}}{T_{e}} \exp \left(-\frac{\varepsilon}{T_{e}}\right),
$$

where $N(\varepsilon)$ is the number of electrons per unit energy per solid angles, $T_{e}$ is the electron slope temperature, $\varepsilon$ is the electron kinetic energy, and $N_{0}$ is total electron number integrated with the energy from zero to infinity per solid angles. The lowest line represents the case of gold foil target $(10 \mu \mathrm{m}$ thickness), and the others are taken at fast ignition shots under the same conditions with Fig. 4. In the result, it can be seen that the emission angle of hot electrons passing through core plasma is smaller than that from gold plane foil. Concretely, in the case of gold foil, the emission angle is about $27^{\circ}$ in half width of half maximum by Gaussian fitting. On the other hand for imploded plasma, this decreases to about $9^{\circ}$. The narrowing can be considered that collimating characteristics of the cone structure ${ }^{4,5}$ or effect of presence of preformed plasma filled in gold cone. ${ }^{6}$

\section{CONCLUSION}

We have developed a multichannel electron spectrometer with a high spatial resolution and wide angular ranges. The error on the electron number and energy is estimated as about $15 \%$ and $5 \%$. The measured electron spectra show the difference of emission angle between foil and imploded cone-shell target, $27^{\circ}$ and near $9^{\circ}$, respectively.

\section{ACKNOWLEDGMENTS}

The authors gratefully acknowledge the support of the Grant-in-Aid for Scientific Research (Contract No. 19206099) and International Collaboration for High Energy Density Science (ICHEDS) of Core-to-Core Program organized by Japan Society for the Promotion of Science (JSPS) and FIREX Project Team.

${ }^{1}$ T. Yabuuchi, A. Das, G. R. Kumar, H. Habara, P. K. Kaw, R. Kodama, K. Mima, P. A. Norreys, S. Sengupta, and K. A. Tanaka, New J. Phys. 11, 093031 (2009).

${ }^{2}$ K. A. Tanaka, T. Yabuuchi, T. Sato, R. Kodama, Y. Kitagawa, T. Takahashi, T. Ikeda, Y. Honda, and S. Okuda, Rev. Sci. Instrum. 76, 013507 (2005).

${ }^{3}$ A. Hiroshi, J. Tomoyuki, and FIREX Project Group, J. Plasma Fusion Res. 81, 98 (2005).

${ }^{4}$ Y. Sentoku, K. Mima, P. Kaw, and K. Nishikawa, Phys. Rev. Lett. 90, 155001 (2003)

${ }^{5}$ H. Habara, K. Adumi, T. Yabuuchi, T. Nakamura, Z. L. Chen, M. Kashihara, R. Kodama, K. Kondo, G. R. Kumar, L. A. Lei, T. Matsuoka, K. Mima, and K. A. Tanaka, Phys. Rev. Lett. 97, 095004 (2006).

${ }^{6}$ T. Tanimoto, A. L. Lei, T. Yabuuchi, H. Habara, K. Kondo, R. Kodama, K. Mima, and K. A. Tanaka, J. Phys.: Conf. Ser. 112, 022095 (2008). 